\title{
Symphony: Simulation, Emulation, and Virtualization Framework for Accurate WSN Experimentation
}

\author{
Laurynas Riliskis, Evgeny Osipov \\ Department of Computer Science, Electrical and Space Engineering \\ Luleå University of Technology \\ 97187 Luleå, Sweden \\ laurynas.riliskis@1tu.se
}

\begin{abstract}
We have developed a simulation framework for testing and validation of WSN applications which closely resembles processes happening inside real equipment including hardware and software induced delays. The core of the framework consists of a virtualized operating system and an emulated hardware platform integrated with a general purpose network simulator ns3. Besides an ability of experimenting with the real code base as in the real deployments our framework allows testing the boundary effects of different hardware components on the performance of distributed applications and protocols. All in all the presented framework allows to substantially shorten the development cycle of WSN applications.
\end{abstract}

Index Terms-wireless Sensor networks, simulation tools, hardware and software modeling

\section{INTRODUCTION}

Traditionally in the area of communication networks simulations is the primary technique for the analysis of performance of various protocols. This is also true in the case of performance studies of wireless sensor networks. WSNs, however, have one important peculiarity, which makes simulation-based studies challenging. Most, if not all, network simulators execute the experiment scenario in high-end machines. In WSNs the resource constrained hardware is in many cases the primary performance limiting factor. Ignoring either the effects of the hardware delays or the particular execution model of operating systems makes the obtained performance figures unrealistic.

The work described in this article is rooted in the authors' own experience in developing and testing a real-life mediumscale distributed WSN application in the domain of intelligent transportation systems [1]. In short the task was to design and deploy a proprietary MAC level protocol and a simple networking application ${ }^{1}$. The final time accounting showed an expenditure of more than 1000 person hours for the whole cycle of the development starting from conceiving the idea until the installation of the fully functioning solution. Remarkably, only $1 / 4$ of the time were spent for the design, simulation based validation and coding for the target operating

\footnotetext{
${ }^{1}$ See http://www.youtube.com/watch?v=GYyWkYfsJhk for a demonstration.
}

system (TinyOS). The other 3/4 of time was consumed by debugging the code on the target hardware and validating the distributed functionality.

This situation is not in any sense unique to our case: Currently several international projects (e.g. WSN-DPMC ${ }^{2}$ ) look at the issues of efficient integrated tools for development of WSN functionality.

In this article we describe a simulation framework, named Symphony, for testing and validation of WSN applications which closely resembles the processes happening inside real equipment including hardware and software induced delays. Symphony consists of three operating and programming scopes: An operating system scope, a hardware scope, and an orchestration and communication scope. The OS scope provides necessary tools and a set of rules for building existing WSN OS (e.g. Contiki, TinyOS, FreeRTOS) to a virtual image. The hardware scope contains a set of models accurately emulating time behavior of hardware components. Network simulator ns-3 offers an orchestration and communication scope.

Besides an ability of experimenting with different applications implemented under different operating systems in one simulation, Symphony offers three unique features for a WSN developer: Experimentation with the real code base as in the real deployments, preservation of the execution model of the underlying operating system and experimentation with the effect of hardware components on the performance of distributed applications and protocols. We conjecture that using Symphony the time for development of a WSN application could be shortened substantially.

The article is organized as follows: In Section II we overview related work in the domain of simulation-based experimentation with WSN. Section III describes the architecture of the framework. Section IV describes result of benchmarking the performance of a network protocol obtained in Symphony, TOSSIM and a testbed. Symphony's technical characteristics

\footnotetext{
${ }^{2}$ WSN DPMC cooperation project. Web portal. Online. Available: http: //www.wsn-dpcm.eu/ .
} 
and scalability issues are analyzed in Section V. Section VI concludes this article.

\section{Related Work in the Operational Scopes of SYMPHONY FRAMEWORK}

Due to straightforward technical, logistical and cost issues experimentation with communication networks is done mainly in simulators. With the emergence of WSN technology the set of existing general purpose network simulators (e.g. ns-2, ns-3, Omnet [2], Qualnet [3], etc.) was extended with WSNspecific frameworks, like Shawn [4] and operating system specific engines such as TOSSIM [5] and COOJA [6]. The main objective with Shawn is to enable testing of abstract algorithms in large scale networks by abstracting away the details of lower-layers' implementation. While not arguing against this objective our work on the Symphony framework targets an orthogonal goal, i.e. to enable large scale realistic simulations with configurable levels of abstraction.

Compared to simulation-based experimentation with highend communication systems two features of WSN technology make its simulations challenging: Delays introduced by lowend hardware components and an execution model of the underlying operating system.

Operating systems for WSN follow three design paradigms: Event-driven (e.g. TinyOS [7]), threaded (e.g. Contiki [8]) and the mixture of the two (see [9]-[11] for a detailed survey of OS for WSN). While there are pros and cons of using each paradigm the fact is that operating system of all three types exist on the market and the performance of the same distributed algorithms and communication protocols could differ drastically depending on the choice of the underlying OS and the composition of the software modules [12].

As the major optimization objective for wireless sensor networks is low-energy consumption all WSN hardware components feature low-energy modes enabling duty-cycling operation. The time delay associated with switching the hardware modes introduces obvious effect on the performance of communication protocols and distributed algorithms in general. This time may vary in the order of magnitude between components produced by different vendors ${ }^{3}$.

TOSSIM is a simulator that accompanies TinyOS. In its essence TOSSIM abstracts away OS specific execution model and substitutes that part of the code with its own run-time environment. Also, the communication protocols on MAC and physical layers are substituted with extremely simplified tablebased communication model. Several TinyOS-specific simulators addressing some inflexibilities of TOSSIM appeared before 2010 (see [13] and reference therein). Most relevant of those are PowerTOSSIM [14], ATEMU [15] and AVRORA [16]: PowerTOSSIM as the name indicates enables the analysis of power consumption properties; ATEMU and AVRORA offer instruction level-emulation of a single hardware platform (Mica2 motes). In addition, only a single application per node

\footnotetext{
${ }^{3}$ For example to turn radio chip on for AT86RF230 (http://www.atmel.com/Images/doc5131.pdf) takes $360 \mu$ while CC2240 (http://www.ti.com/lit/ds/symlink/cc2420.pdf) $1.2 \mathrm{~ms}$
}

is supported. To the best of our knowledge the development of all of them is discontinued.

The work on the integration of TinyOS into OMNeT++ [2] focuses on transforming the $\mathrm{NesC}$ code to $\mathrm{C}++$ classes and running the node as an object in the simulator. According to [17], however, when using this approach the measured performance figures are way too far from those measured in the testbed.

Cooja - a simulation facility for Contiki OS - preserves the OS execution model, which enables instruction level debugging. On the other hand the hardware modeling capabilities are limited to the microcontroller only.

Network simulators ns-2 and ns-3 are the de-facto standard simulation tools in the academic networking research community. Although network simulator ns-3 is the successor to the ns-2 simulator, it is a complete rework of ns- 2 and is not backwards compatible. Ns-3 is a discrete-event network simulator for Internet systems. It contains improvements on the architecture, software integration, and models of the ns-2 simulator. Ns-3 supports multiple radio interfaces per node and features IP addressing, a TCP/IP model that closer resembles the real protocol, and more detailed $802.11 \mathrm{a} / \mathrm{b} / \mathrm{s}$ models. Moreover, ns-3 offers a possibility to run the real implementation of protocols as well as to run simulations in real time.

We conjecture that none of the existing simulators could currently offer the same variety of features and experimentation flexibilities as Symphony. Firstly, we use popular ns-3 simulator as the platform for orchestration and execution of simulation experiments as well as its well established radio propagation models. This choice allows a developer to experiment with holistic machine-to-machine systems featuring heterogeneous radio technologies including communications in backbone networks. Secondly, Symphony features a virtualizer of real operating systems integrated with ns-3 which enables a developer to experiment with different implementations of the same distributed algorithm in the same simulation. Finally, Symphony contains a set of models accurately emulating behavior of hardware components in time and energy domains. All these features allow performing simulations extremely closely resembling the reality.

\section{Symphony - System ARChiteCtURE}

Figure 1 illustrates the core architecture of Symphony consisting of three scopes. The WSN operating system scope deals with the mapping of hardware abstractions in OS to the hardware scope of the Symphony. The hardware scope contains the implementation of hardware models. An XML interface is offered to a user for configuring the models' parameters. The orchestration scope takes care of integrating the OS and the hardware scopes into a holistic simulation environment.

The following subsections elaborate the details of each scope of the Symphony's architecture.

\section{A. WSN Operating System Scope}

The OS scope generates emulated interrupts to OS and calls from OS by tapping into the hardware abstraction layer (HAL) 


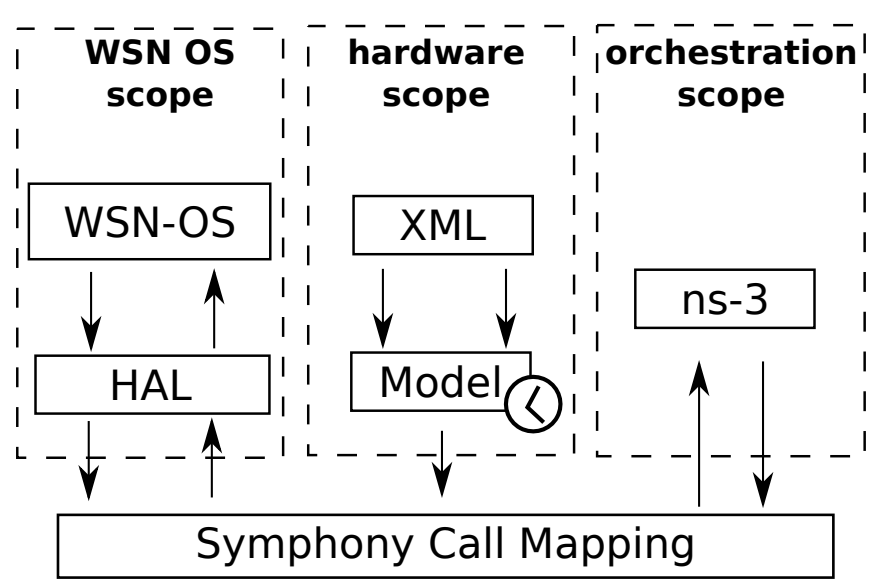

Fig. 1. Architecture of Symphony framework.

of WSN OS as shown in Figure 2. There an operating system makes a call to a hardware element (in this case a call to a radio transceiver to transmit a message). To complete the entire operation it takes time $t_{O S}$ : It is composed of time $t_{H A L}^{p r e}$ for dispatching the call into the underlying HAL; time $t_{H W}^{d}$ is consumed by the device; and time $t_{H A L}^{\text {post }}$ to perform additional operations on the returning path of the call.

Listing 1. A sample of a device model in XML format.

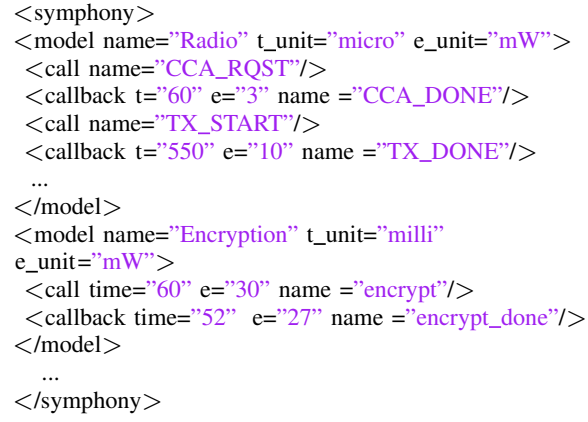

Scalability of simulations heavily depends on the granularity of the underlying simulation model. In order to make Symphony suitable for large variety of scenarios the OS scope of Symphony gives the user a possibility of intercepting calls on different levels of abstraction. The user may choose to remain on the OS level by intercepting calls from OS to HAL and back. In this case the processes inside HAL will be ignored reducing the level of details in the simulation. Alternatively, the user may include HAL functionalities in the simulation for example for debugging purposes. We discuss the effect of the simulations granularity on the performance of Symphony in Section V.

When the desired level of abstraction is selected the user needs to specify the time and energy properties of an operation in the hardware scope of Symphony. After that the execution of operation is appropriately delayed in Symphony's orchestration scope.

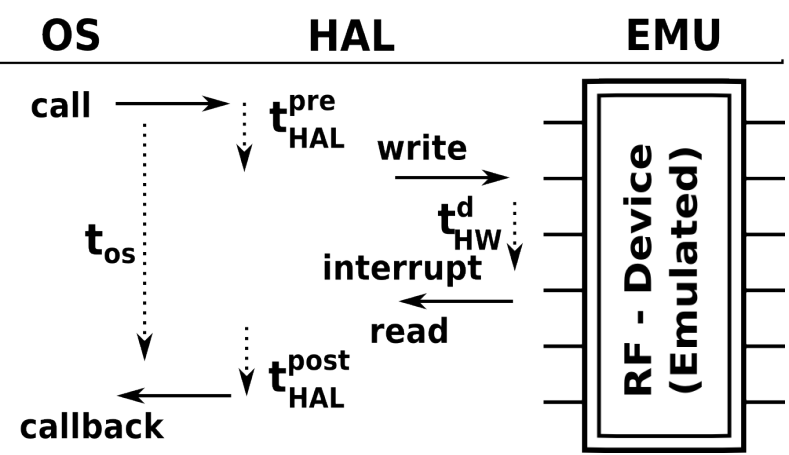

Fig. 2. Time flow within node when sending a packet from application.

\section{B. Hardware Scope}

In Symphony a sensor device is contemplated as a set of models describing in a homogeneous way behaviors of both hardware and software components in time and energy domains. The model of a component defines the time and energy properties when being called (call) as well as when it returns the control to the caller (callbacks). The particular time and energy values are described in the elements' attributes. The model is described using XML format as exemplified in Listing 1 for a hardware component (Radio transceiver) and a software component (Encryption). The model shows for example, that a time it takes from initiating a clear channel assessment (CCA_RQST) until it is done (CCA_DONE) is $60 \mu \mathrm{s}$ and consumes $3 \mathrm{~mW}$ of energy.

The particular values of time and energy attributes are set as result of performing corresponding profiling of the components when performing different operations. The profiling is done by measuring the current flow during operation's execution. An example measurement while performing encryption operation is illustrated in Figure 3 (see a more detailed discussion in Section IV). Some attributes, especially of hardware components, could be taken from a supplied data sheet. The main functionality of the hardware scope, however, is the possibility to experiment with different time and energy characteristic. A set of experiments varying these attributes can be constructed in order to understand the effect of hardware diversity on the performance of distributed applications.

\section{Orchestration and Communication Scope}

The simulation scenarios with Simphony are constructed and executed inside the ns-3 environment. This enables experimentation with complex scenarios reusing native ns3 modules and models. Technically, Symphony adds new type of a node model and the associated infrastructure (containers, helpers, etc.), which are inherited from the base classes of ns-3. From the user perspective, however, the simulation work flow remains the same as in the standard ns-3. This is illustrated in Listing 2 on an example of a simulation with TinyOS operating system. 
Listing 2. Simulation set-up in ns-3 environment.

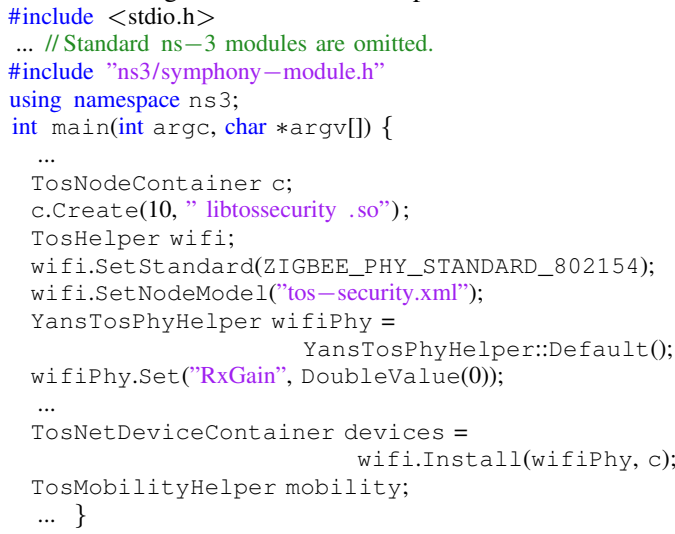

The OS scope is built into a static library and open from within the new node model. When opening the library (line 8 in Listing 2) an XML file of the hardware scope is consulted on which symbols for the callbacks need to be read (line 11 in Listing 2). Behind the scene when initializing the device container (line 16 in Listing 2) the model of the hardware scope is instantiated and initialized with the values from the XML file. This model actually takes care of delaying the execution and book keeping energy properties as described earlier.

One of the biggest technical challenges when implementing Symphony's orchestration scope was overcoming the limitation of Linux on the number of namespaces and the number of static libraries that can be open simultaneously, which is currently set to 14 . As part of the solution we integrated the patched version of an elf-loader [18], which enables loading substantially larger number of node images (limited only by the hardware).

TABLE I

NUMBER OF RECEIVED PACKETS AT THE SINK.

$\begin{array}{lccc}\text { Scheme } & \text { Testbed } & \text { TOSSIM } & \text { Symphony } \\ \text { Plain } & 303 & 336 & 304 \\ \text { CCM } & 127 & 340 & 129 \\ \text { WPI } & 128 & 334 & 130\end{array}$

\section{EXPERIMENTAL SHOW CASE}

In order to demonstrate the unique features of Symphony we selected an example where a computationally heavy encryption function affected the performance of the network protocols, which otherwise is impossible to observe using traditional simulation tools. The results of the performance comparison are shown in Table I. Two algorithms (CCM Counter with CBC-MAC and CSM - cipher-stepping method) providing per-packet Authenticated Encryption with Associated Data (AEAD) were implemented in TinyOS (as part of the separate project). Depending on the security scheme the packets were either authenticated and relayed or first decrypted and then authenticated before they were relayed further. The

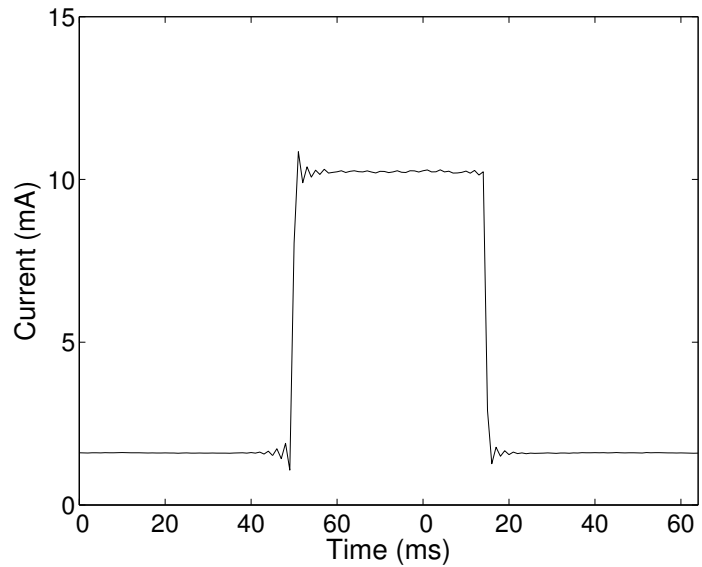

Fig. 3. Energy consumption and time delay when executing AEAD algorithm. National Instrument 4461 ADC (NI-4461-ADC) is used to obtain the current consumption of the node. The measurement board acts as a power supply for the sensor node while at the same time monitoring the time and energy consumption. The PC is used to $\log$ the recorded data.

same implementation was compiled first for the Mulle [19] platform featuring $256 \mathrm{kbit} / \mathrm{s}$ Zigbee transceiver and $16 \mathrm{bit}$ microcontroller, TOSSIM ${ }^{4}$ simulator and Symphony. The test scenario is a chain topology consisting of 10 nodes. The source node generates 35 bytes data packets. Each new packet is generated when the previous one is received by the sink node. For the purposes of this comparison we measure the total number of packets received by the sink node during the test run. Each experiment was repeated ten times and an average number of received packets was calculated. The experiment is then recreated with the same settings in TOSSIM and Symphony. In Symphony we configured the hardware scope with the delay and the current consumption values measured during the execution of AEAD algorithms as well as radio transceiver operations using analog-to-digital converter NI4461-ADC (see Figure 3 for details).

The advantages of Symphony are visible already in the simplest experiment when nodes communicate without AEAD services (marked as "Plain" in the table). Already here TOSSIM gives $10 \%$ more optimistic results compared with the testbed. This is because TOSSIM ignores the delays introduced by hardware when changing the operation modes. More serious problems begin when computationally intensive AEAD operations are enabled. In experiments with the AEAD schemes TOSSIM showed 90 to $100 \%$ erroneous results. These errors are pretty much expected since TOSSIM as well as all other commonly used WSN simulators do not account for the software induced delays. In practice this feature of current simulators would lead to a completely incorrect estimation of an network protocol performances. As we observed in cases with a complex traffic patterns the network exhibits a specific pattern of throughput degradation, which otherwise

\footnotetext{
${ }^{4}$ We selected TOSSIM to enable fair comparison, since plain ns-3 or other conventional simulators would require a simulator-specific implementation of the compared functionality.
} 


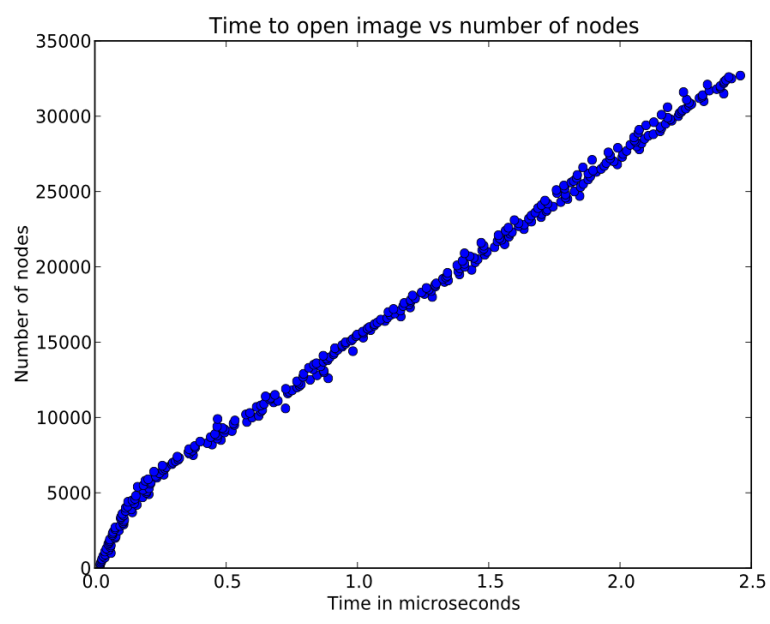

Fig. 4. Time consumed by opening image.

is not visible when running simulations. Due to the limitation on the size of the paper the analysis of these cases will be reported elsewhere. Finally, as predicted, we observe that all results obtained in Symphony are on average $99 \%$ accurate.

\section{Technical Characteristics of Symphony}

The run-time performance of Symphony depends on the mode in which the framework is operating, i.e. either a realtime mode or a virtual time mode. In the case of the virtual time operation the performance of Symphony depends on the desired granularity of the simulation as well as on the complexity of the topology and network scenario. In this article we resort to discussing the runtime performance in real time operation mode only as this mode is one of the features which makes Symphony distinct from other platforms. The assessment of the virtual time operating mode performance of Symphony will be reported elsewhere.

There are two types of operations that consume time during simulation and potentially can affect the simulation accuracy: A library loading and a function call in a virtualized node image. The time for loading many libraries can affect the simulation bootstrapping procedure. Obviously, one needs to wait until all libraries are loaded before starting the simulation. Figure 4 demonstrates the dynamics of this characteristic for different numbers of simulated nodes. As visible from the graph the loading time in scenarios with less then 5000 nodes is practically negligible. After that it grows linearly with the number of nodes. Symphony accounts for this behavior by having a special synchronization function that assures correct start up of the simulations.

In Symphony the granularity of the simulated model is reflected in the size of the compiled library: The lower is the granularity level the large is the size of the library. When operating with large libraries loaded to a simulation in large quantities results to frequent cache misses at CPU level of the host machine during the context switching. This inevitably leads to the increased time for calling any function in a

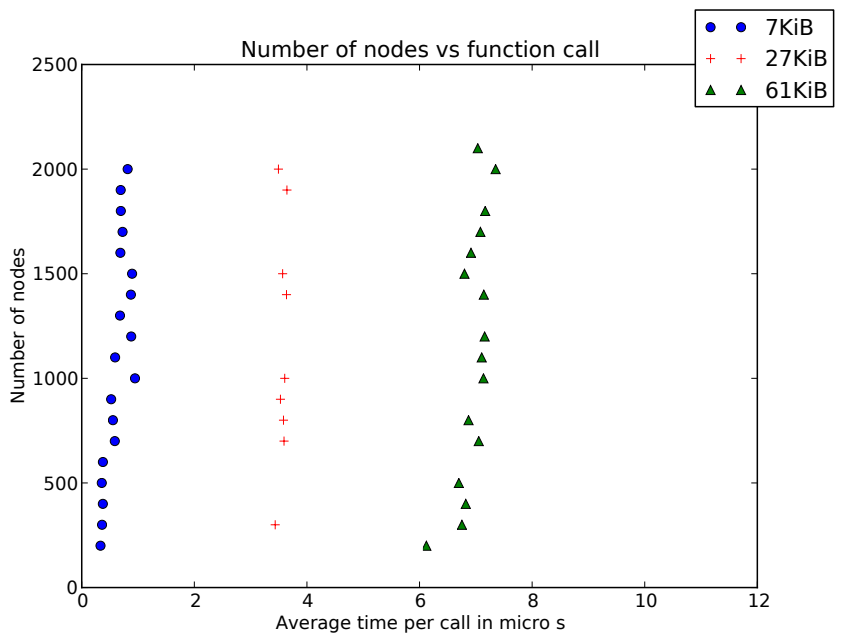

Fig. 5. Function call time. Measured on Intel i7 CPU with 32 GB memory.

particular library. Figure 5 illustrates an average time per call of different size and quantities of nodes in simulation. It shows that the call time only depends on the size of the library and not on the number of nodes for a given size.

This is a hardware imposed limitation. While the accuracy of the experiments performed in the simulated time is not affected by this limitation a user, however, needs to take this delay into account when constructing large scale realtime scenarios. In particular it is essential to ensure that the processing time of an event involving of a execution chain of $n$ calls should be within the real-time constrains of the particular application. In practice one should interpret results in Figure 5 as follows. Assume on a chosen level of granularity the size of the library is $61 \mathrm{KiB}$. Suppose that execution of a operation measured on real hardware takes $20 \mu s$. When configuring this delay in the Symphony's hardware scope one should account for additional $6 \mu \mathrm{s}$ context switching induced delay.

\section{CONClusions AND Future Work}

In this article, Symphony, a framework for realistic WSN simulations is presented. Symphony offers three unique features to a WSN developer: experimentation with the real code base as in the real deployments, preservation of the execution model of the underlying operating system and experimentation with the effect of hardware components on the performance of distributed applications and protocols. The accuracy of the Symphony framework is demonstrated by benchmarking the performance of network protocols measured in a real tested, a conventional WSN simulator and Symphony, where Symphony showed the best match to the real-life results.

\section{REFERENCES}

[1] W. Birk, J. Eliasson, P. Lindgren, E. Osipov, and L. Riliskis, "Road Surface Networks technology enablers for enhanced ITS," in Vehicular Networking Conference (VNC), 2010 IEEE, Dec. 2010, pp. 152-159.

[2] A. Varga and R. Hornig, "An overview of the omnet++ simulation environment," in Proceedings of the 1st international conference on Simulation tools and techniques for communications, networks and systems \& workshops, ser. Simutools '08. ICST, 2008, pp. 60:1-60:10. 
[3] R. Electronics, Online, home page of Qualnet. [Online]. Available: http://www.qualnet.com

[4] A. Kröller, D. Pfisterer, C. Buschmann, S. P. Fekete, and S. Fischer, "Shawn: A new approach to simulating wireless sensor networks," CoRR, vol. abs/cs/0502003, 2005.

[5] P. Levis, N. Lee, M. Welsh, and D. Culler, "TOSSIM: accurate and scalable simulation of entire TinyOS applications,' in SenSys '03: Proceedings of the 1st international conference on Embedded networked sensor systems. New York, NY, USA: ACM, 2003, pp. 126-137.

[6] F. Osterlind, A. Dunkels, J. Eriksson, N. Finne, and T. Voigt, "CrossLevel Sensor Network Simulation with COOJA," in Local Computer Networks, Proceedings 2006 31st IEEE Conference on, Nov. 2006, pp. 641-648.

[7] P. Levis, S. Madden, J. Polastre, R. Szewczyk, K. Whitehouse, A. Woo, D. Gay, J. Hill, M. Welsh, E. Brewer, and D. Culler, "TinyOS: An Operating System for Sensor Networks," in Ambient Intelligence, W. Weber, J. M. Rabaey, and E. Aarts, Eds. Berlin/Heidelberg: Springer-Verlag, 2005 , ch. 7.

[8] A. Dunkels, B. Gronvall, and T. Voigt, "Contiki - a lightweight and flexible operating system for tiny networked sensors," in Local Computer Networks, 2004. 29th Annual IEEE International Conference on, Nov. 2004, pp. 455-462.

[9] M. O. Farooq and T. Kunz, "Operating Systems for Wireless Sensor Networks: A Survey," Sensors, vol. 11, no. 6, pp. 5900-5930, 2011.

[10] A. M. V. Reddy, A. P. Kumar, D. Janakiram, and G. A. Kumar, "Wireless sensor network operating systems: a survey," Int. J. Sen. Netw., vol. 5, no. 4, pp. 236-255, 2009.

[11] J. Yick, B. Mukherjee, and D. Ghosal, "Wireless sensor network survey," Computer Networks, vol. 52, no. 12, pp. 2292-2330, 2008. [Online]. Available: http://www.sciencedirect.com/science/article/ pii/S1389128608001254

[12] C. Margi, B. de Oliveira, G. de Sousa, M. Simplicio, P. Barreto, T. Carvalho, M. Naslund, and R. Gold, "Impact of Operating Systems on Wireless Sensor Networks (Security) Applications and Testbeds," in Computer Communications and Networks (ICCCN), 2010 Proceedings of 19th International Conference on, aug. 2010, pp. 1-6.

[13] M. Imran, A. Said, and H. Hasbullah, "A survey of simulators, emulators and testbeds for wireless sensor networks," in Information Technology (ITSim), 2010 International Symposium in, vol. 2, june 2010, pp. 897 -902 .

[14] V. Shnayder, M. Hempstead, B.-r. Chen, G. W. Allen, and M. Welsh, "Simulating the power consumption of large-scale sensor network applications," in Proceedings of the 2nd international conference on Embedded networked sensor systems, ser. SenSys '04. New York, NY, USA: ACM, 2004, pp. 188-200. [Online]. Available: http://doi.acm.org/10.1145/1031495.1031518

[15] J. Polley, D. Blazakis, J. McGee, D. Rusk, and J. Baras, "Atemu: a fine-grained sensor network simulator," in Sensor and Ad Нос Сотmиnications and Networks, 2004. IEEE SECON 2004. 2004 First Annual IEEE Communications Society Conference on, Oct., pp. 145-152.

[16] B. Titzer, D. Lee, and J. Palsberg, "Avrora: scalable sensor network simulation with precise timing," in Information Processing in Sensor Networks, 2005. IPSN 2005. Fourth International Symposium on, april 2005, pp. 477-482.

[17] U. M. Colesanti, C. Crociani, and A. Vitaletti, "On the accuracy of omnet++ in the wireless sensornetworks domain: Simulation vs. testbed," in Proceedings of the 4th ACM Workshop on Performance Evaluation of Wireless Ad Hoc, Sensor,and Ubiquitous Networks, ser. PE-WASUN '07. New York, NY, USA: ACM, 2007, pp. 25-31.

[18] M. Lacage, "Experimentation tools for networking research," $\mathrm{Ph} . \mathrm{D}$. dissertation, Ph. D. dissertation, Ecole doctorale Stic, Université de Nice Sophia Antipolis, 2010.

[19] Z. Fan, L. Wenfeng, J. Eliasson, L. Riliskis, and H. Mäkitaavola, "TinyMulle: A Low-Power Platform for Demanding WSN Applications," in Wireless Communications Networking and Mobile Computing (WiCOM), 2010 6th International Conference on. IEEE, Sep. 2010, pp. 1-5. 